\title{
Towards Risk Modeling for Collaborative AI
}

\author{
Accepted for presentation at the 1st Workshop on AI Engineering - Software Engineering for AI (WAIN'21) \\ co-located with the 43rd International Conference on Software Engineering (ICSE'21)

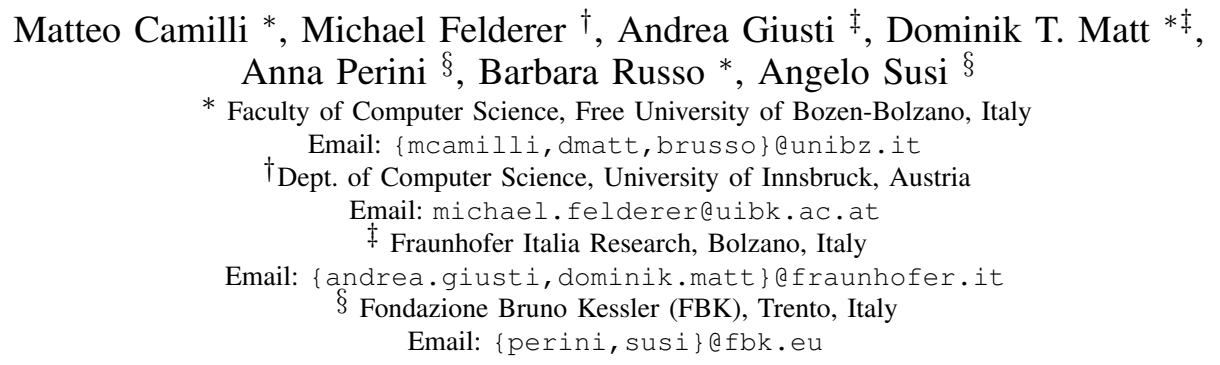

\begin{abstract}
Collaborative AI systems aim at working together with humans in a shared space to achieve a common goal. This setting imposes potentially hazardous circumstances due to contacts that could harm human beings. Thus, building such systems with strong assurances of compliance with requirements domain specific standards and regulations is of greatest importance. Challenges associated with the achievement of this goal become even more severe when such systems rely on machine learning components rather than such as top-down rule-based AI. In this paper, we introduce a risk modeling approach tailored to Collaborative AI systems. The risk model includes goals, risk events and domain specific indicators that potentially expose humans to hazards. The risk model is then leveraged to drive assurance methods that feed in turn the risk model through insights extracted from run-time evidence. Our envisioned approach is described by means of a running example in the domain of Industry 4.0, where a robotic arm endowed with a visual perception component, implemented with machine learning, collaborates with a human operator for a productionrelevant task.
\end{abstract}

Index Terms-Human-robot collaboration, Collaborative AI systems, Risk management, Assurance methods.

\section{INTRODUCTION}

Collaborative AI systems (CAIS) work together with humans in a shared physical space to achieve common goals. Such systems are required to build trust between the human and the machine by ensuring a safe interaction and not prevailing over human needs. CAIS should be flexible to accommodate changing requirements and, at the same time, ensure the satisfaction of key quality criteria for the specific application domain, including appropriate behavior with respect to social rules as well as domain specific standards and laws that are stated by certification and government bodies. Nevertheless, such systems often run in dynamic and uncertain environments that make it difficult to provide strong assurances of acceptable behavior. This condition yields risks of dangerous circumstances because of unfulfilled compliance requirements.

Systematic engineering processes to build and assure that CAIS are in compliance with requirements (e.g., those extracted from human-robot collaboration standards are still

${ }^{1}$ ISO/TS 15066 https://www.iso.org/standard/62996.html); supplementing ISO 10218-1 (https://www.iso.org/standard/51330.html); and ISO 10218-2 on safety (https://www.iso.org/standard/41571.html). immature and require further investigation. Recent surveys report the urgent need for effective engineering processes [1], [2] for "intelligent" components, such as Machine Learning (ML) models, as well as AI systems. In particular, the work presented in [3] discusses challenges and desiderata for assurance methods of such systems. This latter work emphasizes that existing approaches for AI systems are not linked to compliance requirements and possible risks.

This context brought us to reflect on how research in software and systems engineering could contribute to the objective of defining effective methods to build CAIS equipped with ML components (e.g., based on neural networks). Our main focus is on those collaborative systems that heavily rely on ML to implement human perception skills like visual perception, speech recognition, or conversing in natural language under uncertain and evolving circumstances [4]. By collaborating with domain experts, we started to design a risk-driven approach with the objective of supporting the development of ML-equipped CAIS, henceforth referred again to as CAIS for the sake of simplicity.

As part of our ongoing research [5], in this paper we introduce our modeling approach by means of a running example in the context of Industry 4.0. Essentially, we aim at dealing with risk as first class concern. To do so, we propose to extend the RiskML metamodel [6] to include relevant (and uncertain) characteristics of the environment that are perceived by the ML component and possibly expose execution scenarios to risk events. Then, we envision risk-driven assurance methods that provide feedback from empirical evidence collected at run-time, in a closed-loop setting with the world of interest. Finally, assurance methods execute high-risk scenarios and verify semantically meaningful properties to understand whether the run-time behavior of the CAIS is acceptable.

The rest of the paper is organized as follows. In Sect. II] we introduce a running example to put into place major concepts. Then, in Sect. III we elaborate on our envisioned approach. In Sect. IV] we discuss related work. Finally, in Sect. V] we conclude the paper.

\section{A RUNNING EXAMPLE FROM INDUSTRY 4.0}

We illustrate our vision of the problem and the main steps of our approach by means of a running example from the 


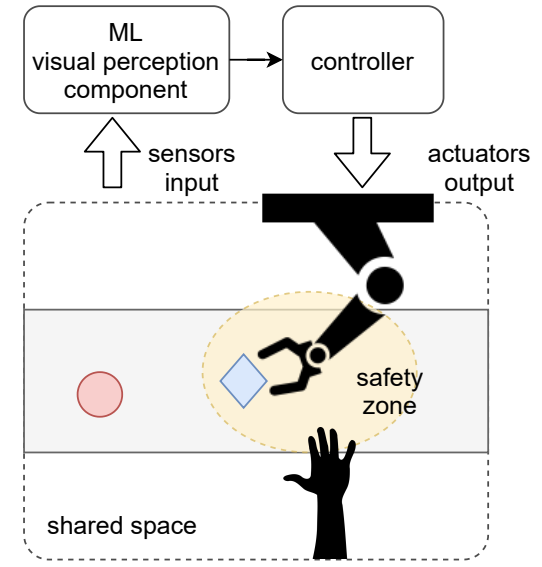

Fig. 1: Collaborative AI running example.

Industry 4.0 domain, borrowed from [7], where a robotic arm collaborates with a human operator for a productionrelevant task. Figure 1 illustrates the running example, where an automated controller of a robotic arm attempts to detect and classify objects (e.g., by color and shape) on a conveyor belt and actuates the proper movements to pick and move the object into the right bucket. The sorting skill is acquired by the robot automatically, based on human demonstrations. The system includes a controller, an actuated mechanical system (i.e., robotic arm), and camera sensors along with a visual perception ML component for classification. This ML component learns on structured heterogeneous data sources associated with features (e.g., shape and color of an object) and yields category labels as output, where labels identify the buckets where the objects must be placed. The operator collaborates with the robot in order to supervise the correct transfer of the desired sorting skill to the robot and can intervene through gestures when corrections are required.

The safety control approach of this example is implemented as in [8], by considering the speed and separation monitoring approach of the standard ISO/TS 15066 for collaborative robotics. Here, a protective separation distance between the human and the robot is checked online using safety zones. The dimension of such zones is dynamically adapted based on the robot motion. Fast motions of the robot can generate safety zones which may be large and therefore negatively affect the realization of collaborative operations. Assuring a successful collaboration in cases in which the robot motions are learnt from humans, as in highly flexible automation, is particularly challenging [9]. In such scenario, we envision a novel risk mitigation process, in which suitable assurance methods are driven by an explicit risk model as described in the following.

\section{OVERVIEW OF THE APPROACH}

In this section, we introduce our envisioned risk mitigation process for CAIS. It contains risk modeling and analysis as well as risk-driven assurance, which provide feedback to each other in order to mitigate known risks below a defined threshold.

\section{A. Risk Modeling \& Analysis}

The risk modeling \& analysis phase is based on the RiskML language [6] that we extend with the notion of domain feature to model aspects of the environment that can be perceived directly by AI components of the system under consideration. Figure 2 shows a model of goals and risks for the collaborative learning example introduced in Sect. $\Pi$ described using the extended RiskML notation. In the model, the concept of Situation is used to model the circumstances under which a certain risk is possible, such as, for example, the situation close interaction. An Event models a change in the circumstances, with a potential negative impact on the goals of the system (e.g., the event insufficient distance). Events have a certain (negative) impact on the affected assets, and they occur with a certain likelihood. The notion of Goal models the desire of a stakeholder in obtaining or maintaining some business value, such as, for example, the goal contact does not happen. The concept of an Indicator embodies a characteristic of a situation, such as, for example, human and robot are in a shared space, as indicator of the situation close interaction.

As mentioned, we finally enrich the RiskML meta-model with the concept of a Domain Feature, which we introduce to capture relevant and quantifiable characteristics of the environment for a given situation. For instance, the ML visual perception component in our example tries to understand what happens in the shared space where a number of aspects are uncertain and vary over time. Such uncertain aspects, influencing the perception capability, shall be explicitly modeled to define the space of possible environment configurations under which the CAIS works. For example, the close interaction situation can lead to risks depending on the luminance of the working area and the speed of the human motion. Thus, domain features yield semantically meaningful inputs for an AI component whose actual values ranges in given domains determined by the context. For instance, the luminance can assume either discrete or continuous values in a given interval that depends on the assumptions about the target production environment.

The model extract in Fig. 2 shows two possible situations: close interaction on the left and online training on the right. The situation close interaction exposes the system to insufficient distance, i.e., a risk event that has an impact on one of the goals of the system, i.e., contact does not happen in this case. The risk can be quantified by verifying the human-robot distance against a given threshold $x$. This impact can compromise the satisfaction of the higher level goals Successful collaboration, compromising in turn the general goal of Trust built. The online training situation exposes the system to poor Al quality, i.e., the risk event that has an impact on the goal accurate learning, which in turn impacts the general goal Human needs respected.

The quantification of risks for a specific situation (e.g., close interaction) is performed on the basis of indicators and domain features during risk analysis. The thresholds and probability distributions of values defined for some (or a combination) indicators and features determine the likelihood of the occurrence of (one or more) risk event(s). As an example the situation close interaction aggregates the values of the dif- 


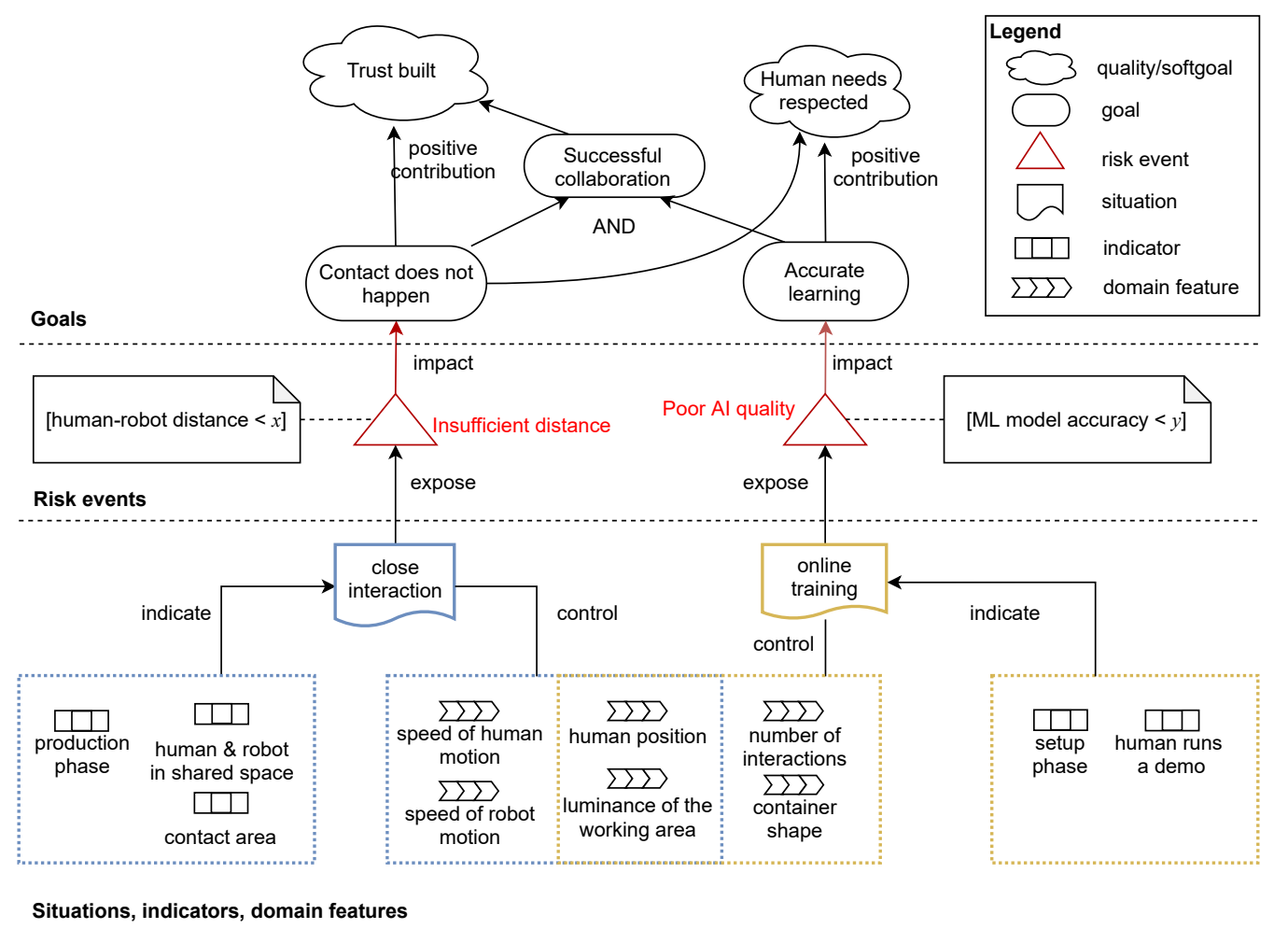

Fig. 2: RiskML model extract of our running example.

ferent indicators (e.g., human and robot in the shared space $=$ True) and from features (e.g., speed of the human motion in and speed of the robot motion in the continuous ranges $[0.0,2.0] \mathrm{m} / \mathrm{s}$ and $[0.5,2.0] \mathrm{m} / \mathrm{s}$, respectively), so recognizing a situation of an active collaboration that may become risky (occurrence of a risk event) when the speed and directions of the human and robot movements lead to violate the safety zone (i.e., the distance became less then $x$ ). In addition, the impact may explicitly be determined or it is considered high for all explicitly modeled events and situations.

The risk analysis drives the assurance process. It is important to point out that thresholds and value distributions are adapted during the assurance, which is described in the next subsection.

\section{B. Risk-driven Assurance}

Our approach maps Situations in the model to compelling assurance cases [3] that expose the system to risk events. Assurance cases are built according to indicators and then executed in order to provide evidence that the run-time behavior is acceptable under such circumstances. In particular, given the inherent infeasibility of "correct by design" AI systems [10], we aim at providing run-time assurances by running the CAIS embedded in a closed-loop setting with a simulated environment (e.g., using GAZEBd ${ }^{2}$ or WeBOts 3 . Thus, an assurance case is simulated multiple times under a number of alternative environment configurations. Such configurations

\footnotetext{
${ }^{2}$ http://gazebosim.org/

3 http://www.cyberbotics.com/
}

are sampled from the search space defined by the set of domain features attached to the corresponding situation. Each individual simulation, can lead to a risk event that is detected by constant monitoring of measurable phenomena, depending on the quantitative constraints attached to risk events. For instance, the event insufficient distance occurs when the distance between the human and the robot is less than $x$, with $x$ determined by the dynamic safety zone, as shown in Fig. 1

Once the domain and range of the relevant domain features are defined by the modeler, we can automatically search for executions that violate model constraints. Since exhaustive enumeration of all possible environment conditions is in general too expensive, we leverage on meta-heuristic optimizing search techniques [11] to guide the sampling towards violations (i.e., minimization of the human-robot distance in our example). Violations of model constraints yield execution scenarios in which the CAIS does not meet one or more goals according to the risk model. In this case, the actual values assigned to domain features can be used to explain the undesired behavior of the system and drive the retraining of the ML component. For instance, low luminance values might lead to decrease the ability to correctly classify the human operator in specific locations of the working area.

As suggested by authors in [10], in addition to metaheuristic optimization, importance sampling techniques (e.g., Bayesian optimization sampling) can be used to update a prior sampling distribution through run-time evidence provided by the execution of the assurance cases. The outcome of this process is a distribution called infill sampling criterion which encourages the important values for the domain features. The 
notion of importance here depends on the target situation exercised by an assurance case. For instance, considering the insufficient distance, important values are likely to minimize the human-robot distance. Such a distribution provides a meaningful feedback to the risk analysis as well as the risk model. For example, sensitivity analysis can be applied to classify the domain features in order to detect the most important factors associated with risks. Furthermore, the highest density region of the infill sampling criterion provides the modeler with the credible intervals of the important values. Loosely, the larger the intervals the higher the likelihood of risks in a given situation. Hence, the magnitude of the credible intervals provides the basis for estimating the probability associated with risk events and thus, close the feedback loop.

\section{RELATED WORK}

Challenges in flexible automation become particularly severe when robotic systems swiftly adapt to different tasks by learning from humans using ML components [12], [9]. Understanding how to properly assure compliance requirements resulting from standards is still an open issue [1], [2], [3].

Risk management have been playing an important role in software and systems engineering processes. The results of risk analysis are typically applied to drive requirements engineering [13] and testing [14] activities. However, risk management processes in the context of CAIS is immature and requires further investigation. The work presented in [15] discusses the risk of susceptible to unintended biases of ML, whereas in [16] authors consider risks for data validation in ML systems. A common risk assessment approach for collaborative robotics applications is based on ISO 12100 [17]. An example of risk assessment process based on this standard is reported in [18]. This latter approach does not take into account AI components embedded into robotic systems.

As stated in [3], assurance methods in the lifecycle of AI systems need a step change. Offline approaches are usually considered more optimistic compared to online approaches [19]. In fact, the online setting aims at generating execution scenarios that might cause violations of requirements by accounting for the closed-loop behavior of AI systems [20]. As advocated in [10], more accurate specifications can be achieved only by considering the execution context (i.e., capturing the model of the environment). Thus, effective assurance methods for CAIS shall should analyze the ML components online, along with their context so that semantically meaningful properties can be verified [10].

\section{Conclusion And Future Directions}

The limited availability of systematic and effective approaches to compliance assurance for CAIS, currently prevents their widespread adoption in different domains such as industrial manufacturing. Domain experts advocate the need of effective risk-driven approaches to build CAIS. In this paper we introduced a high level overview on our modeling approach that considers risk as primary concern with the ultimate objective of building CAIS with strong, ideally provable compliance assurances. As part of our ongoing research, we plan to apply our modeling approach as well as the riskdriven assurance process to build CAIS in real world settings in order to assess applicability and cost-effectiveness by means of empirical evidence.

\section{REFERENCES}

[1] A. Vogelsang and M. Borg, "Requirements engineering for machine learning: Perspectives from data scientists," in 2019 IEEE 27th International Requirements Engineering Conference Workshops (REW). IEEE, 2019, pp. 245-251.

[2] F. Ishikawa and N. Yoshioka, "How do engineers perceive difficulties in engineering of machine-learning systems?-questionnaire survey," in 2019 IEEE/ACM Joint 7th International Workshop on Conducting Empirical Studies in Industry (CESI) and 6th International Workshop on Software Engineering Research and Industrial Practice (SER\&IP). IEEE, 2019, pp. 2-9.

[3] R. Ashmore, R. Calinescu, and C. Paterson, "Assuring the machine learning lifecycle: Desiderata, methods, and challenges," 2019. [Online]. Available: https://arxiv.org/abs/1905.04223

[4] M. Camilli and B. Russo, "Model-based testing under parametric variability of uncertain beliefs," in Software Engineering and Formal Methods, F. de Boer and A. Cerone, Eds. Cham: Springer International Publishing, 2020, pp. 175-192.

[5] M. Camilli, M. Felderer, A. Giusti, D. T. Matt, A. Perini, B. Russo, and A. Susi, "Risk-driven compliance assurance for collaborative ai systems: a vision paper," in Requirements Engineering: Foundation for Software Quality. Cham: Springer International Publishing, 2021, to appear.

[6] A. Siena, M. Morandini, and A. Susi, "Modelling risks in open source software component selection," in Conceptual Modeling - 33rd International Conference, ER 2014, Atlanta, GA, USA. Proceedings, ser. Lecture Notes in Computer Science, vol. 8824. Springer, 2014, pp. 335-348.

[7] A. Giusti, D. Steiner, W. Gasparetto, S. Bertoli, M. Terzer, M. Riedl, and D. T. Matt, "Kollaborative robotik - maschinelles lernen durch imitation," Industrie 4.0 Management, pp. 43-46, 2019.

[8] L. Scalera, V. Di Cosmo, A. Giusti, R. Vidoni, M. Riedl, and D. T. Matt, "Application of dynamically scaled safety zones based on the iso/ts 15066:2016 for collaborative robotics," International Journal of Mechanics and Control, vol. 21, no. 1, pp. 41-49, 2020.

[9] A. Giusti, M. J. A. Zeestraten, E. Icer, A. Pereira, D. G. Caldwell, S. Calinon, and M. Althoff, "Flexible automation driven by demonstration: Leveraging strategies that simplify robotics," IEEE Robotics Automation Magazine, vol. 25, no. 2, pp. 18-27, 2018.

[10] T. Dreossi, D. J. Fremont, S. Ghosh, E. Kim, H. Ravanbakhsh, M. Vazquez-Chanlatte, and S. A. Seshia, "Verifai: A toolkit for the formal design and analysis of artificial intelligence-based systems," in Computer Aided Verification, I. Dillig and S. Tasiran, Eds. Cham: Springer International Publishing, 2019, pp. 432-442.

[11] K.-L. Du and M. N. S. Swamy, Search and Optimization by Metaheuristics: Techniques and Algorithms Inspired by Nature, 1st ed. Birkhäuser Basel, 2016.

[12] A. G. Billard, S. Calinon, and R. Dillmann, "Learning from humans," in Springer handbook of robotics. Springer, 2016, pp. 1995-2014.

[13] A. Cailliau and A. Van Lamsweerde, "A probabilistic framework for goal-oriented risk analysis," in 2012 20th IEEE International Requirements Engineering Conference (RE). IEEE, 2012, pp. 201-210.

[14] M. Felderer, C. Haisjackl, V. Pekar, and R. Breu, "A risk assessment framework for software testing," in International Symposium On Leveraging Applications of Formal Methods, Verification and Validation. Springer, 2014, pp. 292-308.

[15] C. DeBrusk, "The risk of machine-learning bias (and how to prevent it)," MIT Sloan Management Review, 2018.

[16] H. Foidl and M. Felderer, "Risk-based data validation in machine learning-based software systems," in proceedings of the 3rd ACM SIGSOFT international workshop on machine learning techniques for software quality evaluation, 2019, pp. 13-18.

[17] E. ISO, “12100: Safety of machinery-general principles for design-risk assessment and risk reduction (iso 12100: 2010)," 2010.

[18] B. Matthias and T. Reisinger, "Example application of iso/ts 15066 to a collaborative assembly scenario," in Proceedings of ISR 2016: 47st International Symposium on Robotics, 2016, pp. 1-5.

[19] F. Ul Haq, D. Shin, S. Nejati, and L. Briand, "Comparing offline and online testing of deep neural networks: An autonomous car case study," in IEEE 13th International Conference on Software Testing, Validation and Verification (ICST), 2020, p. 85-95. 
[20] A. Gambi, M. Mueller, and G. Fraser, "Automatically testing self-driving cars with search-based procedural content generation," in Proceedings of the 28th ACM SIGSOFT International Symposium on Software Testing and Analysis, ser. ISSTA 2019. New York, NY, USA: Association for Computing Machinery, 2019, p. 318-328. 